\title{
VISUAL CROSS-EXAMINATION OF ARCHITECTURAL AND ACOUSTIC DATA: THE 3D INTEGRATOR EXPERIMENT
}

\author{
L. Bergerot, J.Y. Blaise, A. Pamart, I.Dudek
}

${ }^{1}$ UMR CNRS/MC 3495 MAP,

31 Chemin J.Aiguier, bât. US

13402 MARSEILLE (France)

laurent.bergerot@map.cnrs.fr, jean-yves.blaise@map.cnrs.fr, anthony.pamart@map.cnrs.fr,iwona.dudek@map.cnrs.fr

\section{Commission II}

KEY WORDS: information visualisation, visual metaphors, acoustic data, small-scale architecture, 3D point cloud viewer, multimodal data acquisition, spherical photogrammetry, $360^{\circ}$ camera

\begin{abstract}
:
This paper presents an online application called 3D integrator enabling visual cross-examinations of architectural and acoustic data on web browsers, and explains the rationale behind its development. The tool consists of a series of overlays developed over the Potree 3D point cloud renderer. The 3D integrator is used to display visually, in an interactive environment, the 3D point clouds resulting from a survey protocol tailored to the specific needs of the corpus under scrutiny - fifteen small scale edifices often referred to as "minor heritage assets - and to the research's overall ambition, co-reasoning on architectural and acoustic features at an interdisciplinary level. One of the tool's distinctive features is to project abstract information (acoustic indicators) in a "close to real" 3D space (point clouds), hence merging scientific visualisation and information visualisation practices. The paper shortly sums up the particularities of the survey protocol, and discusses in detail the implementation of the analytical add-ons that have been introduced (visual trace of the survey protocol itself, exploitation of panoramas, volume calculation, and exploratory 3D representation of acoustic indicators). Finally, early uses of the tool are summarised and the services it actually offers at this stage are commented.
\end{abstract}

\section{INTRODUCTION}

This contribution reports on the development of a web-based 3D data visualisation tool, based on the Potree library, and aimed at helping actors to co-examine the architectural and acoustic features of small-scale architectural heritage assets. The tool, named $3 D$ integrator, exploits the results of a specific data acquisition protocol through which metric, visual and acoustic data are co-extracted. Its distinguishing trait is to represent in the $3 \mathrm{D}$ space a set of acoustic indicators (quantitative data resulting from Room Impulse Responses analyses).

The tool's overall design can be summed up as follows:

- A 3D point cloud (resulting from a photogrammetric process) acts as a sort of spatial background for various analytical overlays. In that sense it somehow plays the same role as cartographic layers in leaflet-powered web-based cartographic interfaces.

- Overlays are then added that use the spatial background to position and correlate data: metric, visual, acoustic data are "projected" inside that spatial background.

The approach has been developed in response to a specific set of constraints (lightweight, low-cost solutions, repurposability of outputs, etc. - see section 4). It has been applied to a corpus of 15 rural chapels in south-eastern France, with an ambition to foster repeatability (from acquisition to post-processing steps), and ultimately comparative analyses.

The contribution first positions the development with regards to related research works. The data acquisition protocol is then briefly presented, and its specificities highlighted. The paper's main focus is put on the development of the prototype itself, and on the services expected.

\section{RELATED WORKS}

The prototype is built on top of the Potree 3D point cloud renderer for web browsers (Schütz, 2016), a well-established solution in the application field (see for instance Thivet et al, 2019). The input data results from an $a d-h o c$ survey protocol (see section 3) based on the use of a 360 panoramic camera (a technology discussed in Barazzetti et al., 2018), and on the use of a 3D microphone (Figure1, b), the mhacoustics em32 Eigenmike microphone (a spherical array of 32 microphones relevant for sound field analysis and for sound perception studies as demonstrated in Farina et al., 2011).

The strategy adopted is to introduce a series of JavaScriptpowered overlays on top of Potree. They are used to trigger userside actions (volume calculation for instance) or to project abstract information in the 3D space. One of the main originalities of the research probably lies in the effort made to bridge two sub-disciplines of the "data visualisation" community:

- Scientific visualisation, where what is primarily seen relates to, and represents visually a physical "thing" (Spence, 2001).

- Information visualisation, where one deals with abstract information, and produces graphics aimed at information seeking tasks (Kienreich, 2006). In that sub-discipline 3D shapes are most often visual metaphors (see examples in Aigner et al., 2011).

In the prototype we present, two "3D metaphors" are used to convey an abstract information (quantitative acoustic data, namely C50 clarity and PWD indicators). These metaphors do not resemble "real" acoustic instruments. But their position and the graphical coding used (colours corresponding to frequencies, quantities, and time frames) are indeed in direct relation with the spatial and temporal acoustic behaviours of "real" spaces.

\section{DEVELOPED METHODOLOGY}

The prototype's development, and the services it is intended to deliver, are closely related to a specific, interdisciplinary survey protocol co-designed by architects and acousticians (see 
Acknowledgements). Further detailed in Blaise et al., 2020, it combines existing techniques, and can be summed up as follows:

- Two self-levelling laser levels are used that project laser beams on surfaces of the building (Figure1, a).

- A systematic spatial grid comprising four speakers and four microphones is positioned with regards to the chapels' original function (celebrant vs. listener opposition).

- Intersection of the laser beams on surfaces (Figure1, c), and position of the eight components of the grid, are acquired using a rangefinder that outputs DXF files.

- The acoustic survey consists of the recording on each of the four microphone positions of a sine sweep emitted from each of the fours speakers (several times iteratively in order to spot and eliminate "outliers").

- The photogrammetric acquisition is carried out with a 360 panoramic Camera positioned on each tripod forming the grid, and then moved in different positions decided in situ (pyramidal sequence in each position).

The 3D Integrator aggregates heterogeneous outputs of the survey step and of the post-processing chain: raw photographic material, panoramas, XYZ coordinates of the spatial grid (DXF files), 3D point clouds (including localisations of the grid of acoustic devices), room impulse responses, auralizations calculated for the 4 points recording points (MA, MC, MD,MG and quantitative acoustic indicators.

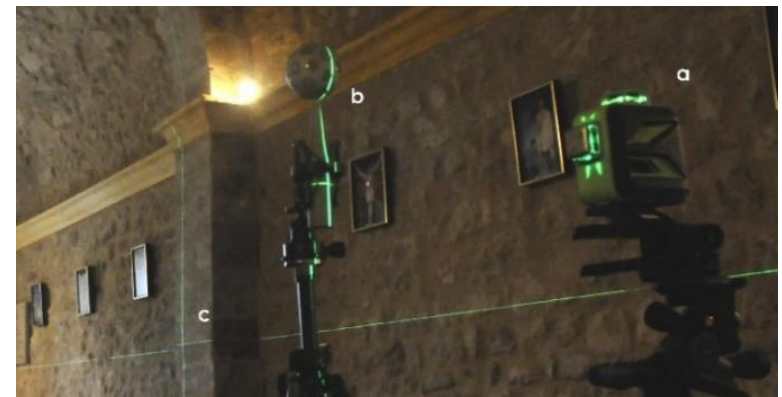

Figure 1: The Eigenmike spherical array of 32 microphones (b), aligned vertically on the survey protocol's spatial grid, and an intersection of laser beams used for the scaling step (c).

The pyramidal data acquisition protocol (see Figure 4) consists of an overlapping block of 5 pictures for each tripod position (instead of a single one) in order to i) cover more quickly the space to survey hence to speed up data capture and ii) densify and improve the panoramic-based camera network. The shooting sequence was eased and time saved by the use of a rotating levelarm tripod coupled with panoramic head. This protocol is also meant to slightly improve data accuracy optimizing the unstitched image quality (composed of highly distorted low-cost double fisheye camera) by orienting the lens axis to all coplanar surfaces (floor/ceiling, longitudinal walls, and transversal walls) according to the architectural indoor space. A data-quality evaluation and comparison including terrestrial laser scanning as reference (TLS) has been performed and is provided in (Blaise et al. 2021). Our results are aligned with previous works assessing the potential of fisheye and spherical photogrammetric application for scanning indoor and narrow spaces, namely a fast and efficient solution for coarse 3D reconstruction which was completely consistent and compliant to our purposes.

The resulting point cloud is converted for Potree, and the rest of the process consists in developing JavaScript-powered overlays that project pieces of data and information in the 3D space. The 3D integrator is basically an enhanced Potree interface: in addition to the native $3 \mathrm{D}$ point cloud visualisation and manipulation tools, a selection menu (top bars) allows users to select one out of the fifteen buildings studied, and specific tools are added to Potree's native toolbar (positioned on the left side). When relevant the selection of one of these specific tools triggers the displaying of a sub-menu (typically user configurations) on the right side (Figure 2).

These tools and sub-menus are part of the JavaScript-powered overlay that has been developed, as well as the scientific and information visualisation solutions developed for the project.

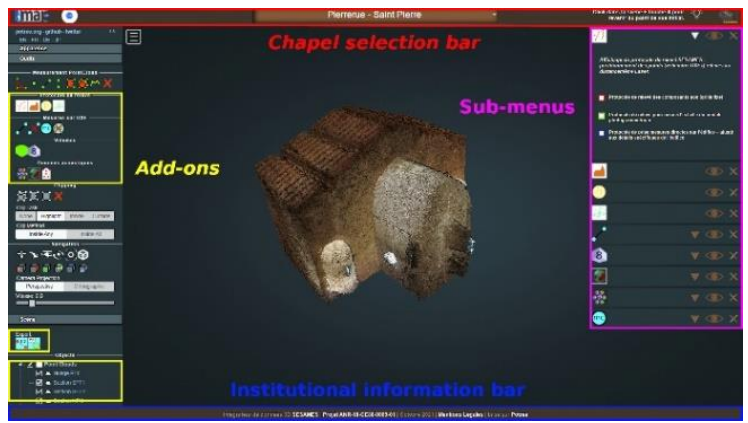

Figure 2: Overview of the enhanced interface with selection menu, institutional information, tools and sub menus.

This development required no less than 10,000 lines of code, not including PHP requests. The Potree renderer is used to display a complete point cloud, but also allows users to display only subclouds. These sub-clouds are slices calculated externally and then integrated into the application. Their purpose is to facilitate the reading of the data (avoiding occlusions when observing the whole 3D scene), typically DXF paths, spheres representing panoramas, etc. In most of the figures presented in this article, such sub-clouds selections are represented.

\subsection{Visualisation of the survey's raw outputs}

The first add-ons introduced focus on simply viewing the data resulting from the survey protocol itself, for each chapel. This includes the DXF input extracted from the rangefinder (represented as polylines, cf. Figure 3) and thumbnail images associated with each DXF point (these images are automatically recorded by the rangefinder distortion camera all along the protocol). This input acts as control points during the photogrammetric reconstruction used to scale and orient the model. The data set is composed of three DXF files representing respectively the acoustic devices survey protocol ( 9 points), the photogrammetric model scaling survey protocol (12 points), and the direct building measurement protocol - a protocol adjusted in situ in order to capture specific architecture details (between 12 points and 20 points).

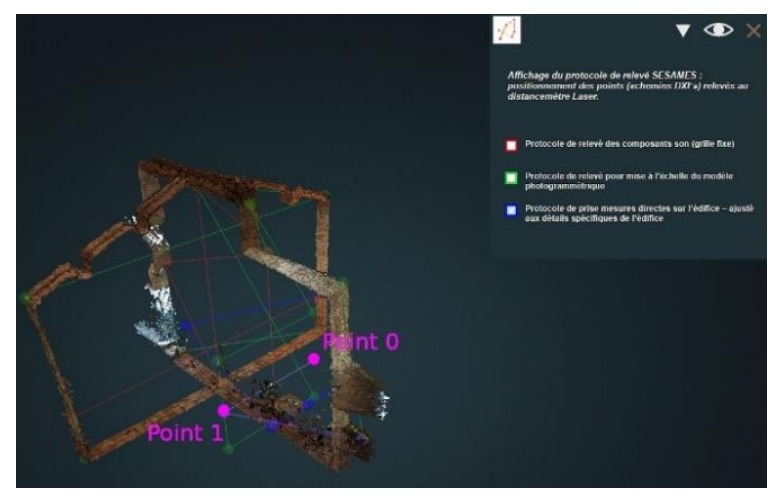

Figure 3: the three polylines corresponding to the three DXF files : acoustic devices (red), scaling of the model (green) and direct measurements (blue). 
The data resulting from the survey protocol also includes the panoramas extracted from the 360 camera, which are materialised in the 3D integrator by spheres (XYZ positions extracted during the photogrammetric processing). These spheres are positioned according to the aforementioned pyramidal data acquisition protocol (Figure 4). The intersection between the mouse pointer and the $3 \mathrm{D}$ spheres is obtained using the raytracing method, hence clicks on these spheres can be detected. Clicks on spheres give access to online immersive panoramas viewed using the panolens.js library. These panoramas include auralizations (simulations of how one same soundtrack would be perceived if played in the various chapels). They act as a general public end product useful for local communities in charge of preserving the buildings and enhancing their value.

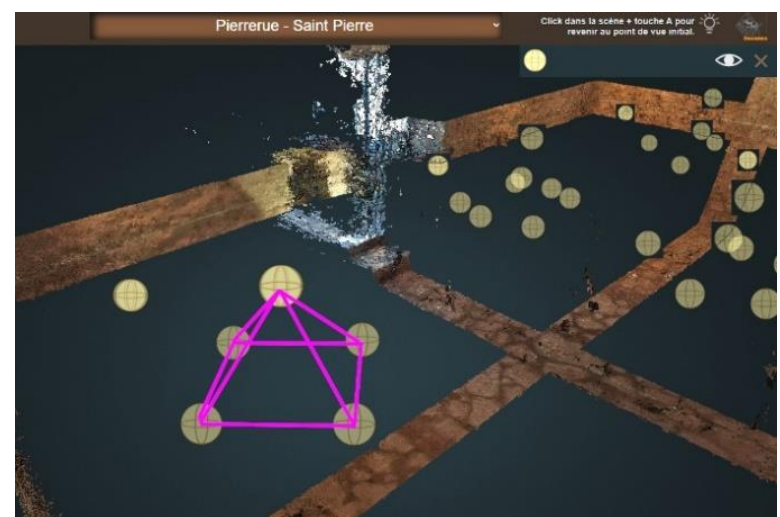

Figure 4: View of the spheres, in yellow, allowing access to the panoramas, arranged according to the pyramidal survey protocol shown in purple.

\subsection{Measurements and naming (DXF points)}

Other specific modules or add-ons allow users to manipulate the survey data. The first one provides users with means to perform on-the-fly measurements on the aforementioned DXF points (native Potree tools only allow the calculation of distances on the point clouds). The pointing of DXF points is done with the same method used for panoramas, ray tracing.

The module enables an on-the-fly displaying of the distance between two points (Figure 5), and can calculate distances in sequence or separately. Another module has also been added to Potree through which measurements can be saved locally or online in JSON format. Measurements made by one user can then be re-used outside the integrator or re-loaded into it via the corresponding sub-menu.

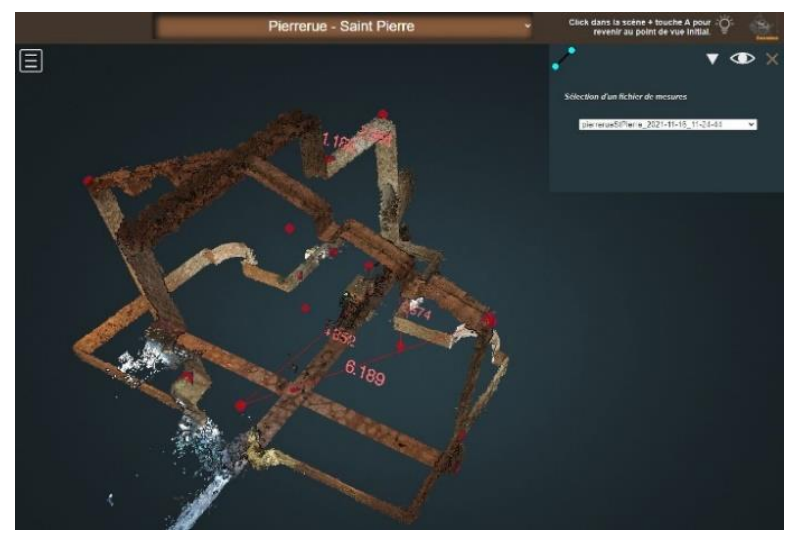

Figure 5: Example of measurements made on the DXF data
Another add-on allows the naming of DXF points (Figure 6). We needed this module because the points in the DXF files are not named, and accordingly cannot be identified and associated unambiguously with the systematic identifiers used in the survey protocols (ec, eg, cc, ....) when displayed in the 3D integrator. As for the access to panoramas and measurement calculations, this naming is done by choosing a DXF point and using the raytracing method. A point can then be named using the sub-menu corresponding to the tool. This sub-menu also allows to edit an erroneous name. As for measurements, a module has been added to the Potree menu which allows the names of the points and their coordinates to be saved in JSON format, so that they can be redisplayed in the integrator at a later date.

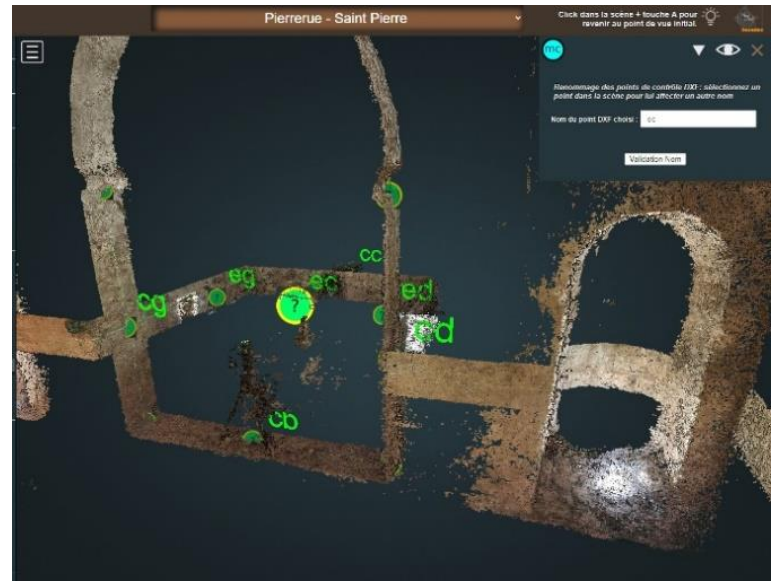

Figure 6: Example of naming of DXF points in progress, here point ec is being edited

\subsection{Visualisation of the laser beams}

As mentioned earlier, the survey protocol bases on the use of two self-levelling laser levels that project green laser beams on surfaces of the building, with intersections of these beams acting as control points for the photogrammetric processing step.

A visualisation tool has also been developed thanks to which laser beams are materialised as green-coloured sub-clouds, thereby showing the setting of the survey with regards to the interior spaces of each chapel (Figure 7).

To do so we use the dense point cloud and then cut it on the $X$, $\mathrm{Y}, \mathrm{Z}$ axes, according to the positions of the laser levels, in order to keep only a "thin green slice" of the point cloud, materialising the trace of the laser levels' beams. The chapel's dense point cloud, loaded in addition to the basic point cloud, is necessary in order to display sufficient information.

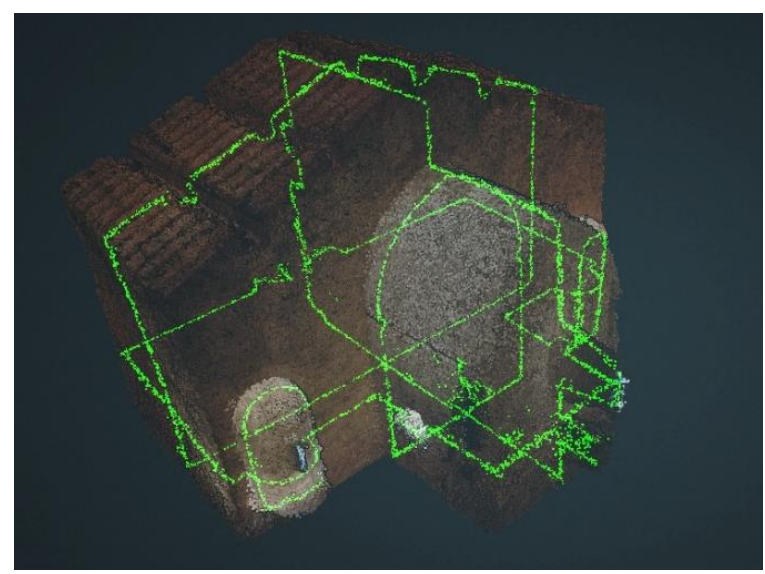

Figure 7: Visualisation of the laser levels' beams 


\subsection{Volume calculation}

Two add-ons have been developed in order to calculate and to visualise approaching volumes of the interiors, also using the dense point clouds: voxel-based volume estimation, and convex hull method.

3.4.1 Voxel-based volume estimation: This first solution allows a representation (based on an on-the-fly calculation) of the approaching volumes of the chapels by voxel-based segmentation of the dense point cloud.

The aim of this voxel-based calculation is to have a quantified volume, approximate but calculated in the same way for all the chapels, according to a given level of segmentation, in order to carry out relative analyses and to establish a possible architectural volume-sound relationship in the whole corpus.

To obtain this type of volume, we proceed as follows: after calculating the bounding box of the point cloud, it is cut into voxels on the $\mathrm{X}, \mathrm{Y}$ and $\mathrm{Z}$ axes, in as many parts as are indicated in the tool's sub-menu. This process is relatively long because, for example, cutting the bounding box into 24 parts, i.e. $24 * 24 * 24$, makes 13824 voxels to be checked!

The first step consists of processing the voxels composing the surface of the point cloud. For each voxel, we look if points of the point cloud are present in it. If so, we record the coordinates of the voxel processed and also assign to it the value 1 to establish that this voxel is part of the point cloud surface.

Using that data, recorded in a table, we can define minimum and maximum $\mathrm{X}, \mathrm{Y}, \mathrm{Z}$ values for each axis processed - in other words the minimum/maximum values for boundaries of the interior space. We thus obtain a first table of the dimension of the number of processed voxels, with values 0 for voxels without points or not yet processed and values 1 for those containing points.

Once this is done the so-called interior voxels are processed, i.e. those contained in the point cloud. The set of voxels that do not have a value of 1 is browsed and, based on the minimum and maximum values calculated previously, two tests are carried out for each axis, i.e. six tests ( $\mathrm{X} \min , \mathrm{X} \max , \mathrm{Y} \min , \mathrm{Y} \max , \mathrm{Z} \min$ and $\mathrm{Z} \max$ ) to determine whether the voxels processed are within these limits. If this is the case, we indicate with the index 2 (for interior) that the treated voxel is inside, otherwise we leave the index value at 0 .

We thus obtain a second table with all the voxels, their coordinates and an index $(0,1,2)$ allowing to know its status (external, surface or internal). The table can then be used as input for the visualisation of the volumes (Figure 8).

A sub-menu allows users to manipulate this volume after calculation. Thanks to sliders, they can play on the $\mathrm{X}, \mathrm{Y}$ or $\mathrm{Z}$ axes to display more or less voxels on the three axes. Two options can be used to visualise the surface voxels (in purple) and/or the interior voxels (in yellow). The outer voxels are never shown. An indication of the approximate volume, calculated from the sum of the volumes of the surface and interior voxels, is also available for comparative analysis.

3.4.1 Convex hull method volume estimation: this second solution allows the representation of the approaching volume of the chapels by modelling the point clouds' convex hull. It proposes another view of the approaching volume. This alternative solution did not require any particular algorithm, it relies on a function named ConvexHull already existing in the ThreeJS library, also used in this program. Again this function required the dense point cloud, as for the representation of the laser levels and the approximating volume per voxel, for a correct calculation of the convex envelope.

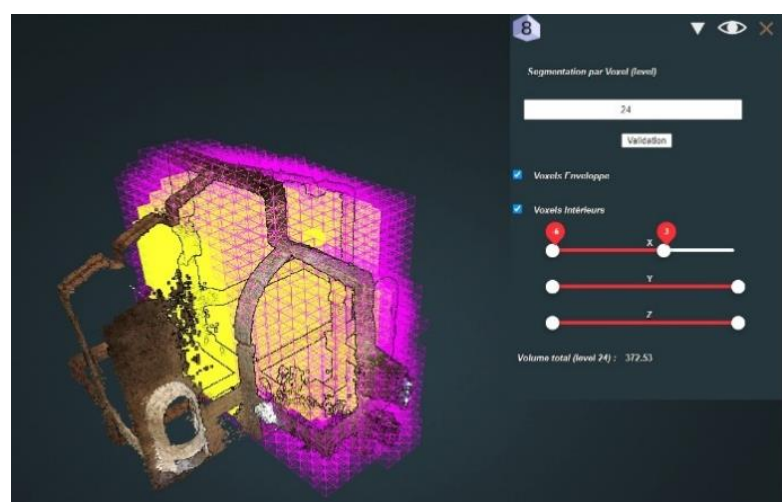

Figure 8: This representation was obtained by creating a bounding box around the whole cloud, then by cutting it on the 3 axes, in a predetermined number of cuts, here 24 , then by eliminating all the voxels which are outside of the point cloud. A distinction is made between the surface voxels and those of the interior. On this example is shown the manipulation of this voxel division via a menu on the $\mathrm{X}, \mathrm{Y}$ and $\mathrm{Z}$ axes (right part, submenu). In purple surface voxels, in yellow, the interior voxels. The current user manipulation is done on the $\mathrm{X}$ axis.

\subsection{Visualisation of acoustic indicators}

Two different add-ons have been developed in order to project in the 3D space acoustic indicators, the values of which are represented using abstract visual metaphors. The first one displays information on the C50 clarity indicator, the other represents values of an indicator calculated using the "plane wave decomposition" method mapped visually as a sort-of 3D energy map.

3.5.1 Visualisation of the 32-channel C50 clarity indicator: This indicator (early/late impulse response ratio) is visualised using a sort-of "garlic flower visual metaphor" for each frequency $(125,250,500,1 \mathrm{~K}, 2 \mathrm{~K}, 4 \mathrm{~K}$ and $8 \mathrm{~K})$ and for each transmitter-receiver pair (Figure 9).

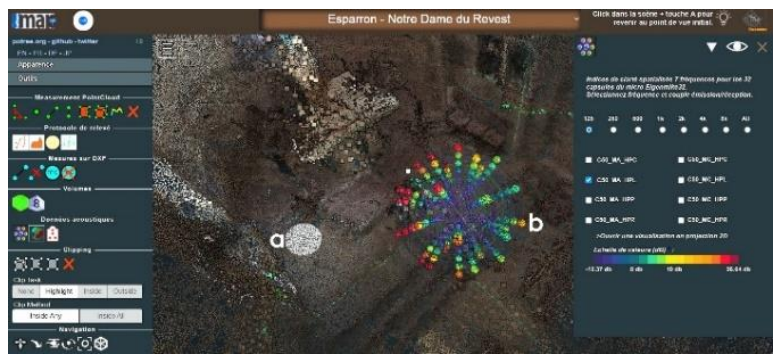

Figure 9: Visualisation of the 32-channel C50 clarity indicator for the left emission point (white sphere in a). Concentric coloured spheres convey three pieces of information: quantitative value (colour), angular position, and frequency. Unsurprisingly high values occur closer to the emitting point, but in (b) an outlier remains a question mark at analysis time.

For each chapel chosen, the creation of the metaphor begins with the reading of the MA and MC positions of the Eigenmike 32 microphone recorded for each chapel.

Then for all the acoustic data, read from eight files representing the eight transmitter-receiver pairs, a calculation of the minimum and maximum values is performed, followed by a calculation of the spherical coordinates in order to position each capsule and by a calculation of the colour of each of the 32 capsules according to the minimum and maximum values previously mentioned. 
For each metaphor, a white sphere representing the emission point (the position of the emitting loudspeaker) has also been represented.

These metaphors can then be manipulated thanks to the sub-menu visible on figure 9 , on the right, allowing to manage the frequency to be displayed, to see all the frequencies at the same time as well as the choice of the transmitter-receiver pair. A coloured bar gives an idea of the scale of acoustic values represented in these metaphors.

3.5.2 Visualisation of the PWD indicator: this indicator (a cartography of energy calculated with the "Plane Wave Decomposition" method) is represented by spherical heat maps. This visualization allows to see the distribution of the data over 360 degrees, using a colour scale that helps the analysts localise in space trends and exceptions. But when talking about acoustic data, temporal aspects play a major role. The tool allows users to interactively display nineteen time frames (i.e. nineteen successive maps) per transmission-reception pair, and to switch between transmission-reception pairs. Shortly said this is used to better understand how this energy indicator moves and decreases in space and time (Figure 10).

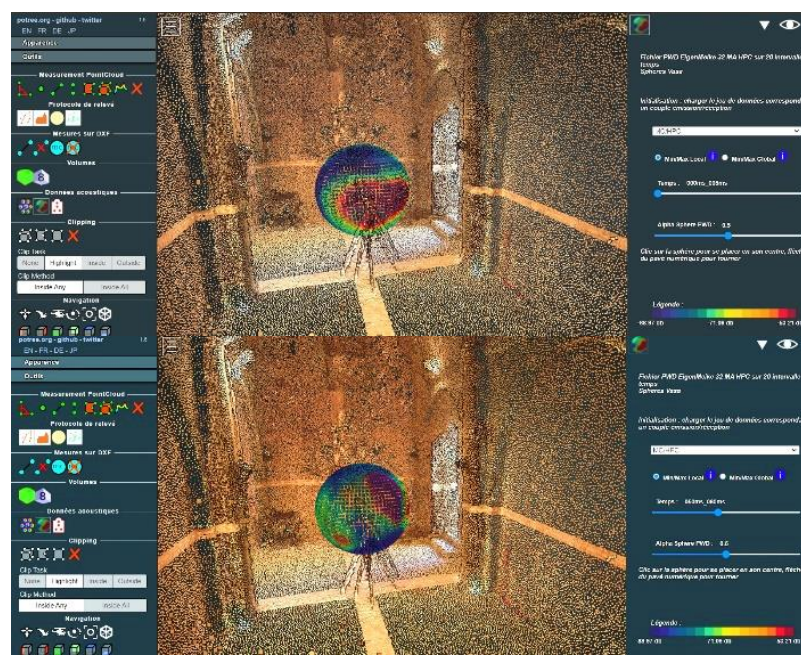

Figure 10: Visualisation of the PWD indicator for a sound emitted on the axis of a chapel, and corresponding to two time frames (top, 0 to 0,005 milliseconds, bottom, 0,05 to 0,06 milliseconds). Note the evolution of the pattern over time, and the dissymmetry pattern (high values in dark red). In that specific case they could ascribable to an uneven distribution of openings.

The display of the visualisation is made possible by the following method: after reading values for each transmission-reception pair, they are placed in a three-dimensional table (time, row number, and column number). The various pairs are processed as the user chooses them in the sub-menu. The data is contained in nineteen files, representing the nineteen time intervals or time frames, each file having a matrix of 37 rows (vertical angular division) by 72 columns (horizontal angular division), i.e. a total of 404,928 values for PWD acoustic indicator for each chapel! The minimum and maximum values are then calculated, according to the user's choice in the sub-menu, either in a socalled global manner, i.e. including all the time intervals, or in a so-called local manner, i.e. only on the chosen time interval.

A colour scale has then been established and allows each value to be coloured by transposing it into a wavelength, within a given interval, limited by these maximum and minimum values previously calculated and also transposed. Then, according to the position of these values in the matrix, they are placed in 3D space thanks to the calculation of spherical coordinates, on the surface of the visual metaphor. All these operations allow the spherical heat map to be represented as a sphere of coloured sprites, each sprite representing a value in the matrix. The sub-menu linked to the tool allows users to navigate in the various time frames thanks to a time slider.

Again, as with the clarity indicator visualisation, the emission sphere is represented as a white sphere.

Users can also get inside the spherical heat map by clicking on it (using the ray-tracing method mentioned above and moving the user's point of view), and display the panorama corresponding to the position being analysed, in order to appreciate the evolution of the acoustic indicator with respect to the architectural space and to time.

\subsection{Development difficulties and constraints}

The development of the 3D integrator raised some difficulties due to the constraints of the project (a challenging corpus, an original survey protocol) and to the development for web browsers.

First of all, due to the heaviness of some data (dense point clouds in particular) and some calculations (volume per voxel), the application undergoes certain slowdowns depending on its use. This is visible when choosing a chapel and loading the corresponding data one, which can take up to one minute. It was necessary to develop asynchronous functions, so that the data, sometimes heavy, is loaded successively and in the right order, which adds to the slowness of the application.

The architectural particularities of some of the interiors that we have surveyed have sometimes been constraining, forcing us to recalibrate some data, in particular the DXF.

On the other hand, the specifications of modules having evolved during the project, it was necessary to satisfy new requests progressively, which sometimes required a partial or complete rewriting of some functions. This was particularly the case for acoustic data, an issue that was raised at half-way of the integrator's development. The use of the tool by project members has also made its ergonomics evolve to meet new requirements. Thus, the sub-menus mentioned earlier in this article have been implemented for each of the integrator's tools to facilitate their use. An offline mode has also been created. More generally, this development feeds an open debate on how to provide local actors or citizens with means to collect and exploit such datasets in the context of poorly funded, unprestigious, minor heritage assets (low-cost survey protocol, analytical overlays inside a web browser, with the corresponding limitations to expect at this stage). Yet this debate goes far beyond the scope of this paper.

\section{RESULTS}

The prototype is used at this stage to extract general dimensions that serve as a basis for proportion-as-ratios (Cohen, 2014) comparative analyses (on the whole or parts of buildings, and across the collection of buildings). It is also used to extract specific dimensional features called segments that serve as a basis for the visualisation and sonification of the rhythmic of the interiors (helping to read the alternation of shapes and sizes along cross-sections). But naturally its core role is to help the actors involved (architects and acousticians) to cross-examine data sets and acoustic behaviours of comparable, small-scale architectural interiors. These three usage scenarios are briefly exemplified below. Yet It is important to stress that the tool has not been designed in order to promote and instrument acoustic simulations per se : this is a different issue, and not one that has been picked up in the context of this research. 
In addition, the 3D integrator aims to contribute to one of the research axis of the SESAMES project focusing on data traceability, reproducibility of methods and repeatability of processes. The data display through the $3 \mathrm{D}$ integrator also act as a representation of the multimodal data acquisition protocol itself, operated on-site. In that sense, the integrator displays the raw data and helps the interpretation of the processed data, but it is also a means to keep a trace of the instrumental set-up and of the survey strategy applied (and adapted) for each chapel. This is the reason why the viewer also integrates all the information needed to document and make others understand how the data were acquired, including elements such as DXF polylines and linked pictures used to point instrument positions and architectural dimensions, intersections of the levelling laser beams, spherical camera positions reflecting the photogrammetric network. This is also expressed by the choice of surveying the space with the instruments installed on measurement points whilst the laser beams turned on instead of a prioritising on a clean and faultless $3 \mathrm{D}$ reconstruction of the indoor space.

A lot remains to be done in the interpretation of the observations the prototype helps making, but it does offer an original service, and one targeted to a corpus with severe self-imposed operational constraints: a lightweight instrumentation (remote sites with accessibility issues, poor funding), a fast contact-free survey protocol (3 hours as a maximum, all included), no power supply in situ, a setup that should be reproduced identically across the whole collection (comparability issue), congested interiors, varying lighting conditions, varying architectural layouts adapted to the analysis of divergent compositional patterns, etc.

\subsection{Extraction of dimensional characteristics}

The 3D integrator is developed in the context of a research programme, a major objectives of which is to compare (formally and acoustically) buildings that are supposedly somehow "similar" (in terms of original function, general architecture, and scale). Obviously, significant dimensions (length, width, height, in overall and in parts) are among the variables the need to be correlated. To do this, a systematic dimensional analysis grid was first established which identifies for each building the dimensions to be extracted on three axes. The simple case illustrated below (Figure 11) gives an idea of the different analysis filters used to extract the dimensional characteristics: distinction nave / chancel, characteristics specific to each bay, each transverse arch, data related to the covering, etc.
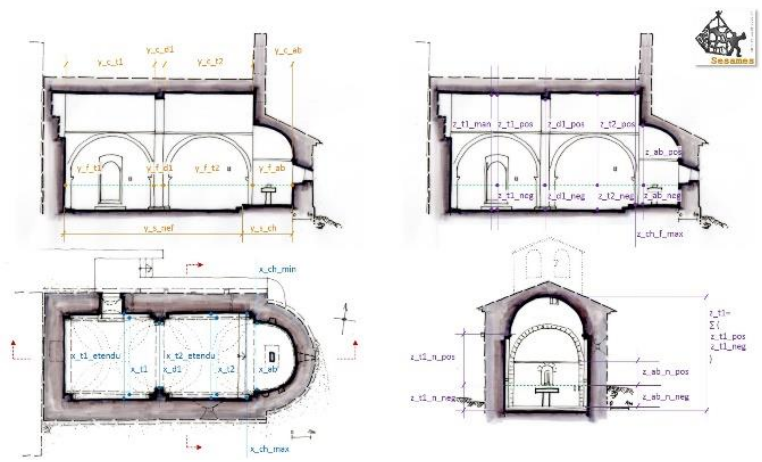

Figure 11: An illustration of some of the predefined "significant dimensions" the values of which can be extracted using the 3D Integrator.

The 3D integrator displays previously oriented and scaled point clouds: it can therefore be used to directly extract the dimensional characteristics corresponding to the systematic dimensional analysis grid (on the three $\mathrm{X}, \mathrm{Y}, \mathrm{Z}$ axes). This extraction is manual: picking of points in the $3 \mathrm{D}$ scene, possible export to JSON format. The benefit of this approach is that it allows the user to adapt the measurement according to the quality of the point cloud and with regards to the position of disruptive elements (furniture typically). It obviously also introduces a degree of uncertainty in the extradition. The development of an automated or semi-automated procedure for extracting these dimensions is part of the project's prospects, but the difficulties to be expected should not be underestimated: the interiors of the buildings studied are, in some cases, very congested (rood screen, high altar, baptismal font, pulpit, bas-relief, and of course, paintings or ex-votos). Accordingly this indeed is a research line, but it goes far beyond the objectives we set ourselves in developing the 3D integrator.

For each building 50 dimensions (on average) were extracted using the 3D integrator's functionalities. Some of these dimensions correspond to distances measured in situ "traditionally", using a Leica A8 rangefinder. This allows a qualitative (and rough) assessment of the reliability of the dimensional characteristic extracted from the integrator. The quality of the measurement is impacted by the user's picking, the granularity of the measurement allowed by the integrator $(1 \mathrm{~cm})$, the differences in density and quality within a point cloud and between point clouds, but above all by factors related to the measurement itself and its post-processing, upstream of the integrator, a point commented in (Blaise et al. 2021).

Of the fifteen buildings concerned, the discrepancies between the measurements extracted via the 3D integrator and the control data taken in situ with rangefinder are in the worst cases (poorly defined cloud) of up to $3 \%$ (3 centimetres per metre) and are generally between 0.5 and $1 \%$ ( 0.5 to 1 centimetre per metre). This result is compatible with the objective of the development: to provide a light-weight method of extracting dimensional characteristics for comparative analysis in an architectural study. It would naturally not be compatible with a metrological monitoring of buildings.

The dimensional features extracted thanks to the 3D integrator were used at this stage for two separate studies, the first focusing on the notion of proportion (Quote), the second with that of "contour", briefly summarised in the following section.

\subsection{Sonification of dimensional characteristics}

In this experiment we reinterpret a generic model that we had developed in another context to analyse moulded architectural elements (Blaise and Dudek, 2012). In a nutshell, this model aims at representing cross-sections in the form of sequences of segments, segments to which are associated qualitative indicators (type of material for example). This codification is intended to highlight the rhythms of an architectural profile, a recurrence of patterns, etc.

This model is based on two essential notions: control points (starting and ending points of the segments) which generally correspond to breaks in continuity in the geometry of the form (e.g. angle) and segments which characterise a path (between their two control points) and are most often associated with a single support object (a wall, a vault, etc.). "What happens" between the two control points is documented in several ways, typically geometry of the segment, name, material, etc.

In the framework of the SESAMES project, the original formalism is no longer applied to moulded architectural elements (capitals, arches, architraves, etc.) but to the overall plan (contour) of a building in which bays, double arches, apses, etc. alternate (Figure 12).

The 3D integrator is used here to extract control points for each segment, basing on the green trace left in the 3D point cloud by 
the laser beams projected by the self-levelling laser levels (hence ensuring horizontality and verticality). Once each segment has been identified and documented (dimensions and qualitative indicators), it is possible to transfer the values of each segment in the form of a 'sound' and, in fine, to propose a 'sonification' of the contour, in other words a sound mapping of the architectural information. This exploratory work is carried out by a research laboratory partner of the project, the PRISM research unit, which is developing complementary sonification strategies using the data extracted via the $3 \mathrm{D}$ integrator (an experiment that falls outside the scope of this article)

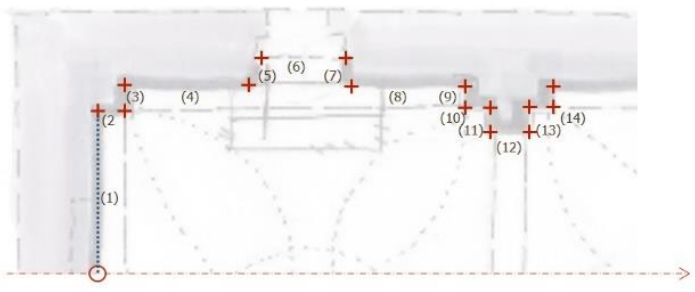

Figure 12: Control points between segments forming a contour .

\subsection{Spatialisation and visualisation of acoustic indicators}

In addition to allowing the extraction of dimensional characteristics, the $3 \mathrm{~d}$ integrator has the particularity of projecting acoustic indicator values into the $3 \mathrm{D}$ space. These values are mapped to two original "visual metaphors", respectively named allium 32 (C50 clarity indicator) and Vasa sphere (PWD indicator), whose construction is detailed in the previous section.

Our use of the term 'visual metaphor' here is open to discussion: W. Kienreich (Kienreich, 2006) defines it as using a form of representation based on a real-world equivalent to display information. A typical example of a visual metaphor is the "information landscape" proposed by V.Sabol (Sabol, 2012) to allow users to visually locate groups of thematically related documents, grouped to form "hills" within an artificial landscape. The two visualisations proposed are not, strictly speaking, equivalents of a real object, or that of the sensor whose data they exploit, the 32-channel eigenmike spherical microphone.

But on the other hand, both visualisations are positioned in the 3D space at the exact point of recording, and their shape (spherical) evokes this of the microphone: this is why we have preferred to use this expression.

It is important to mention that both visualisations provide an indication about values of acoustic indicators: they do not position "raw data" in the 3D space but the result of a postprocessing of the impulse response, which can be seen as a filtering made to highlight different acoustic characteristics.

The "Allium 32" visualisation builds on the geometry of the microphone (32 channels) and delivers quantitative information by frequency or all frequencies combined, for 8 pairs of emission points / collection points (see section 3). By moving around the visual metaphor in $3 \mathrm{D}$ space, the user can observe the variations in value for each sector and each frequency.

However, this possibility of establishing a link in space between the value of the indicator and the architecture of the building comes at a cost: the user can only observe one side at a time, and does not see all the values at once. This is therefore a departure from one of the golden rules of a good visualization as coined by E.R. Tufte (Tufte, 2001): enforce comparisons within the eyespan. The effects of occlusion and the cognitive impact of the user's need to find his way in the 3D space space are among the important reasons why InfoVis solutions make little use of the third dimension, or if so under system-guided conditions.
The Allium 32 visual metaphor displayed in the 3D Integrator is therefore complemented with a $2 \mathrm{D}$ visualisation using the same indicator values, but allowing the whole set of values to be observed at once (Figure 13). What is lost in terms of spatialization is gained in terms of completeness and term-byterm comparison.

The PWD indicator translates the 2664 values calculated for this indicator (one value every five degrees) into the form of a sphere made up of small coloured surfaces, for each pair of emission point / collection point, as mentioned in section 3 .

Here the values do not correspond to a frequency, but to an instant: for each pair of emission point / collection point of each building, 19 tables are actually produced, each containing 2664 values. This visualisation is coupled to a time slider that allows users to browse through these time-framed indicator values, in the form of 19 time slots. These slots have growing durations in order to maximise the readability of the phenomena to be observed (significant events appearing much more closely together at the beginning than at the end of the sequence).

However, as for 3D InfoVis solutions, InfoVis solutions including a time slider-like temporal navigation tool (sometimes called "dynamic visualisations" in the literature) certainly provide an additional service but have a service with a cost: the user can only observe one moment at a time, and cannot see all the values at once.

The 3D heat map visualisation is therefore complemented by a 2D solution that we have called eGrid, which uses the same indicator values, but allows the user to observe all the values at once in the form of visual grids corresponding to the tables of values. In the version of this visualization illustrated below, all the values for a point of capture are visualized, thus the values corresponding to the three points of emission, and the various time slots (Figure 13).

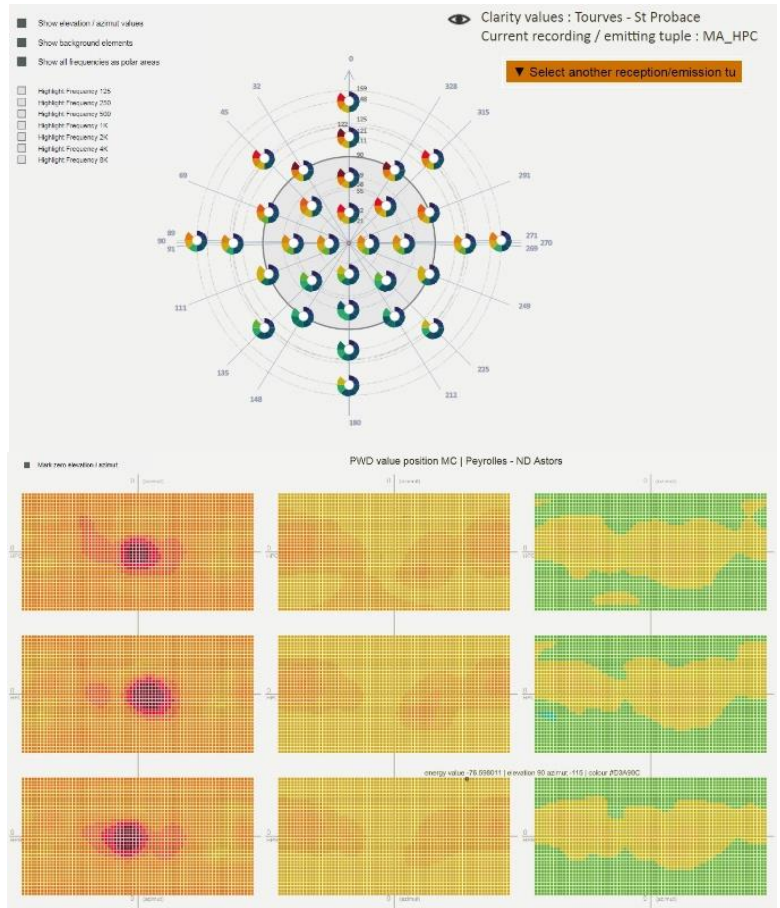

Figure 13: 2D equivalents of the solutions designed in the context of the 3D Integrator: (top) the Allium 32 2D visualisation, with each sphere corresponding to one of the 32 capsules of the microphone (here projected on a flat surface, somehow like a map) and (bottom) a partial view of the eGrid visualisation (only three time frames, and three emission/reception points are shown). 


\section{CONCLUSIONS}

This research is conducted by a multidisciplinary team pulling together architects and acousticians (see section Acknowledgements), and on a quite specific corpus the impact of which proved prominent. Indeed, the "3D integrator" has not been designed as (and is not) a general purpose $3 \mathrm{D}$ point cloud visualisation platform. Our approach is not intended to explore purposes of 3D point cloud analysis (issues such as segmentation, classification, see for instance Grilli et al., 2017) but focuses on enabling a better understanding of the multimodal data sets collected, with as core motivation trying to enhance their intelligibility.

Said briefly, its role is to help actors reason on the correlation of an acoustic behaviour with architectural features. It plays the role of an infovis solution, where the visualisation of data sets acts as food for thinking and reasoning, in trial and error processes targeting a fine grain interpretation of the data.

A number of open issues (in terms of methods or of technology) can be debated in the light of this experiment such as limitations of a 3D visualisation per se in terms of information readability and navigability, challenges concerning comparison enhancement inside 3D settings, scalability, response time, export formats, etc. The usage scenarios tested up to now show the development is promising, feeds interdisciplinary discussions on practical cases, but also that the attractiveness of a 3D tool should not distract analysts from digging into their data in many other ways.

\section{ACKNOWLEDGEMENTS}

The 3D Integrator builds on the results of a survey protocol that was co-designed, and implemented, with the PRISM research unit (UMR 7061 PRISM, Aix Marseille Univ / CNRS / MC https://www.prism.cnrs.fr/).

The PRISM research unit also provided the 3D Integrator's development team with fruitful insights on the nature of the acoustic data and indicators concerned by the experiment. Authors are particularly thankful to A. Vidal, S. Fargeot, M. Aramaki, S. Ystad, R. Kronland-Martinet.

The project is funded by the ANR, the project-based funding agency for research in France, under the id ANR-18-CE38-000901 .

\section{REFERENCES}

Aigner, W., Miksch, S., Schumann, H., Tominski, C. 2011. Visualization of Time-Oriented Data. Human-Computer Interaction Series, Springer-Verlag, London, 2011.

Barazzetti, L., Previtali, M., Roncoroni, F. 2018. Can we use lowcost 360 degree cameras to create accurate $3 \mathrm{~d}$ models? ISPRS TC II Mid-term Symposium “Towards Photogrammetry 2020", Riva del Garda, Italy, 4-7 June 2018. https://doi.org/10.5194/isprsarchives-XLII-2-69-20, pp 69-75.

Blaise, J.Y, Dudek, I., Pamart, A., Bergerot, L., Vidal, A., Fargeot, S., Aramaki, M., Ystad, S., Kronland-Martinet, M., 2020. Space \& sound characterisation of small-scale architectural heritage: an interdisciplinary, lightweight workflow. International Conference on Metrology for Archaeology and Cultural Heritage 2020 https://halshs.archivesouvertes.fr/halshs-02981084

Blaise, J.Y, Dudek, I., Pamart, A., Bergerot, L., Vidal, A., Fargeot, S., Aramaki, M., Ystad, S., Kronland-Martinet, M., 2021. Acquisition \& integration of spatial and acoustic features: a workflow tailored to small-scale heritage architecture. ACXTA-IMEKO Journal (in press)

Blaise, J.Y, Dudek, I., Identifying and Visualizing Universal Features for Architectural Mouldings. IJCISIM International Journal of Computer Information Systems and Industrial Management Applications, Vol. 4, 2012, ISSN2150-7988, pp. $130-143$

Cohen, M.A. Conclusion: Ten Principles for the Study of Proportional Systems in the History of Architecture. Architectural Histories, 2(1), 2014. DOI: http://doi.org/10.5334/ah.bw.

Farina, A., Amendola, A., Capra, A., Varani, C. 2011. Spatial analysis of room impulse responses captured with a 32-capsule microphone array. 130th Audio Engineering Society convention, London, 2011.

Grilli, E., Menna, F., Remondino, F., 2017. A review of point clouds segmentation and classification algorithms. ISPRS Archives Volume XLII-2/W3, doi:10.5194/isprs-archives-XLII2-W3-339-201.

Kienreich, W., 2006. Information and knowledge visualisation: an oblique view, MiaJournal vol 0.

Schütz, M., 2016 Potree: Rendering Large Point Clouds in Web Browsers. PhDThesis Vienna University of Technology.

Sabol, V., 2012. Visual Analysis of Relatedness in Dynamically Changing Repositories. Coupling Visualization with Machine Processing for Gaining Insights into Massive Data. http://www.gamsau.map.cnrs.fr/modys/abstracts/Sabol.pdf

Spence, R., 2001. Information Visualization Addison Wesley.

Thivet, M., Verriez, Q., Vurpillot, D., 2019. Aspectus: a flexible collaborative tool for $3 \mathrm{D}$ data exploitation in the field of archaeology and cultural heritage In Situ Vol. 39 (19) https://doi.org/10.4000/insitu.21240

E.R Tufte, E.R. 2001. Visual explanations Graphics Press, Cheshire.

See:

http://anr

sesames.map.cnrs.fr/viewer_ply_dxf/SESAMES_Integrateur/pro grammes/SESAMES_Integrateur.html 